\title{
Intelligent Drive Assistance System
}

Anuja Kale ${ }^{1}$, Aditya Raut ${ }^{1}$, Swati Sinha ${ }^{2}$

1Student, Department of Information Technology, Vidyalankar Institute of Technology, Mumbai Maharashtra, India ${ }^{2}$ Professor, Department of Information Technology, Vidyalankar Institute of Technology, Mumbai Maharashtra,

India

\section{Article Info}

Volume 7, Issue 3

Page Number: 96-104

\section{Publication Issue :}

May-June-2021

\section{Article History}

Accepted : 08 May 2021

Published : 13 May 2021

\section{ABSTRACT}

The report proposes the research conducted and the project made in the field of computer engineering to develop a system for driver drowsiness detection to prevent accidents from happening because of driver fatigue and sleepiness. The report proposed the results and solutions on the limited implementation of the various techniques that are introduced in the project. Whereas the implementation of the project give the real world idea of how the system works and what changes can be done in order to improve the utility of the overall system. Furthermore, the paper states the overview of the observations made by the authors in order to help further optimization in the mentioned field to achieve the utility at a better efficiency for a safer road. A person driving needs to be able to focus on driving at all instances. Any prolonged or sudden complications to the person driving the vehicle can cause serious accidents/damages. To ignore the importance of this could result in severe physical injuries, deaths and economic losses. Road incidents remain the leading type of fatal work-related event, carrying tremendous personal, social, and economic costs. While employers with a fixed worksite can observe and interact directly with workers in an effort to promote safety and reduce risk, employers with workers who operate a motor vehicle as part of their job have fewer options. Drowsiness detection system is regarded as an effective tool to reduce the number of road accidents. This project proposes a non-intrusive approach for detecting drowsiness in drivers, using Computer Vision. Developing various technologies for monitoring and preventing drowsiness while driving is a major trend and challenge in the domain of accident avoidance systems. This project proposes a non-intrusive approach for detecting drowsiness in drivers, using Computer Vision. Developing various technologies for monitoring and preventing drowsiness while driving is a major trend and challenge in the domain of accident avoidance systems. Haar face detection algorithm is used to capture frames of image as input and then the detected face as output.

Keywords : Face Detection, Haar, MAR, Drowsiness Detection 


\section{INTRODUCTION}

Humans have always invented machines and devised techniques to ease and protect their lives, for mundane activities like traveling to work, or for more interesting purposes like aircraft travel. With the advancement in technology, modes of transportation kept on advancing and our dependency on it started increasing exponentially. It has greatly affected our lives as we know it. Now, we can travel to places at a pace that even our grandparents wouldn't have thought possible. In modern times, almost everyone in this world uses some sort of transportation every day. Some people are rich enough to have their own vehicles while others use public transportation. However, there are some rules and codes of conduct for those who drive irrespective of their social status. One of them is staying alert and active while driving. Neglecting our duties towards safer travel has enabled hundreds of thousands of tragedies to get associated with this wonderful invention every year. It may seem like a trivial thing to most folks but following rules and regulations on the road is of utmost importance. While on road, an automobile wields the most power and in irresponsible hands, it can be destructive and sometimes, that carelessness can harm lives even of the people on the road. One kind of carelessness is not admitting when we are too tired to drive. In order to monitor and prevent a destructive outcome from such negligence, many researchers have written research papers on driver drowsiness detection systems. But at times, some of the points and observations made by the system are not accurate enough. Hence, to provide data and another perspective on the problem at hand, in order to improve their implementations and to further optimize the solution, this project has been done.

Our current statistics reveal that just in 2015 in India alone, 148,707 people died due to car related accidents. Of these, at least 21 percent were caused due to fatigue causing drivers to make mistakes. This can be a relatively smaller number still, as among the multiple causes that can lead to an accident, the involvement of fatigue as a cause is generally grossly underestimated. Fatigue combined with bad infrastructure in developing countries like India is a recipe for disaster. Fatigue, in general, is very difficult to measure or observe unlike alcohol and drugs, which have clear key indicators and tests that are available easily. Probably, the best solutions to this problem are awareness about fatigue-related accidents and promoting drivers to admit fatigue when needed. The former is hard and much more expensive to achieve, and the latter is not possible without the former as driving for long hours is very lucrative. When there is an increased need for a job, the wages associated with it increases leading to more and more people adopting it. Such is the case for driving transport vehicles at night. Money motivates drivers to make unwise decisions like driving all night even with fatigue. This is mainly because the drivers are not themselves aware of the huge risk associated with driving when fatigued. Some countries have imposed restrictions on the number of hours a driver can drive at a stretch, but it is still not enough to solve this problem as its implementation is very difficult and costly.

The main sections of viola jones is listed below:

- Integral Image allowing speedy evaluation of the features.

- Classifier function built on a few features.

- Combining the classifiers in a cascade structure increases the speed of the detector as it focuses on regions of interest.

\section{LITERATURE SURVEY}

Ngxande, Mkhuseli, Jules-Raymond Tapamo, and

Michael Burke. "Driver drowsiness detection using behavioral measures and machine learning techniques: A review of state-of-art techniques." 2017 
Pattern Recognition Association of South Africa and Robotics and Mechatronics (PRASA-RobMech). IEEE, 2017.

\section{Jabbar, R., Al-Khalifa, K., Kharbeche, M.,} Alhajyaseen, W., Jafari, M. and Jiang, S., 2018. Realtime driver drowsiness detection for android application using deep neural networks techniques. Procedia computer science, 130, pp.400-407. Kumari, Jyoti, R. Rajesh, and K. M. Pooja. "Facial expression recognition: A survey." Procedia Computer Science 58 (2015): 486-491.

Ngxande M, Tapamo JR, Burke M. Driver drowsiness detection using behavioral measures and machine learning techniques: A review of state-of-art techniques. In2017 Pattern Recognition Association of South Africa and Robotics and Mechatronics (PRASA-RobMech) 2017 Nov 29 (pp. 156-161). IEEE. Bhoi, N. and Mohanty, M.N., 2010. Template matching based eye detection in facial image. International Journal of Computer Applications, 12(5), pp.15-18.

Sharafi, Z., Soh, Z. and Guéhéneuc, Y.G., 2015. A systematic literature review on the usage of eyetracking in software engineering. Information and Software Technology, 67, pp.79-107.

Azim, T., Jaffar, M.A., Ramzan, M. and Mirza, A.M., 2009, December. Automatic fatigue detection of drivers through yawning analysis. In International Conference on Signal Processing, Image Processing, and Pattern Recognition (pp. 125-132). Springer, Berlin, Heidelberg.

\section{Kaplan, Sinan, Mehmet Amac Guvensan, Ali Gokhan} Yavuz, and Yasin Karalurt. "Driver behavior analysis for safe driving: A survey." IEEE Transactions on Intelligent Transportation Systems 16, no. 6 (2015): 3017-3032.

\section{Young, Kristie, Michael Regan, and Mike Hammer.}

"Driver distraction: A review of the literature."

Distracted driving 2007 (2007): 379-405.

\section{PROBLEM STATEMENT}

Fatigue is a safety problem that has not yet been deeply tackled by any country in the world mainly because of its nature. Fatigue, in general, is very difficult to measure or observe unlike alcohol and drugs, which have clear key indicators and tests that are available easily. Probably, the best solutions to this problem are awareness about fatigue-related accidents and promoting drivers to admit fatigue when needed. The former is hard and much more expensive to achieve, and the latter is not possible without the former as driving for long hours I s very lucrative.

\section{PROPOSED SYSTEM}

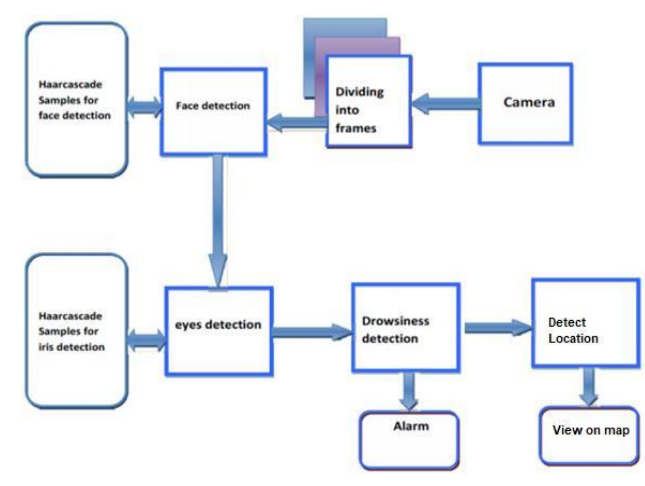

Fig 1- Proposed System Architecture

Fig 1. showcases the various important blocks in the proposed system and their high level interaction. It can be seen that the system consists of 5 distinct modules namely:

- Video acquisition

- Dividing into frames

- Face detection

- Eye detection

Drowsiness detection. In addition to these there are two external typically hardware components namely, Camera for video acquisition and an audio 
alarm. The functionality of each these modules in the system can be described as follows:

Video acquisition: Video acquisition mainly involves obtaining the live video feed of the automobile driver. Video acquisition is achieved, by making use of a camera.

Dividing into frames: This module is used to take live video as its input and convert it into a series of frames/images, which are then processed.

Face detection: The face detection function takes one frame at a time from the frames provided by the frame grabber, and in each and every frame it tries to detect the face of the automobile driver. This is achieved by making use of a set of pre-defined Haarcascade samples.

Eyes detection: Once the face detection function has detected the face of the automobile driver, the eyes detection function tries to detect the automobile driver's eyes. This is achieved by making use of a set of pre-defined Haarcascade samples.

Drowsiness detection: After detecting the eyes of the automobile driver, the drowsiness detection function detects if the automobile driver is drowsy or not, by taking into consideration the state of the eyes, that is , open or closed and the blink rate.

Location Tracking : When the driver is detected drowsy, we calculate the latitude and longitude of the area in which the driver is present at that time and store the latitude and longitude co-ordinates in the database server. Using the longitude and latitude coordinates we can track the location of the driver where he is drowsy on the map

\section{IMPLEMENTATION}

\section{Video Acquisition}

OpenCV provides extensive support for acquiring and processing live videos. It is also possible to choose whether the video has to be captured from the inbuilt webcam or an external camera by setting the right parameters. As mentioned earlier, OpenCV does not specify any minimum requirement on the camera, however OpenCV by default expects a particular resolution of the video that is being recorded, if the resolutions do not match, then an error is thrown. This error can be countered, by overriding the default value, which can be achieved, by manually specifying the resolution of the video being recorded.

EYE_AR_THRESH $=0.3$

EYE_AR_CONSEC_FRAMES $=30$ YAWN_THRESH $=20$

\section{Dividing into frames}

Once the video has been acquired, the next step is to divide it into a series of frames/images. This was initially done as a 2 step process. The first step is to grab a frame from the camera or a video file, in our case since the video is not stored, the frame is grabbed from the camera and once this is achieved, the next step is to retrieve the grabbed frame.

While retrieving, the image/frame is first decompressed and then retrieved. However, the two step process took a lot of processing time as the grabbed frame had to be stored temporarily. To overcome this problem, we came up with a single step process, where a single function grabs a frame and returns it by decompressing.

\section{Face detection}

Once the frames are successfully extracted the next step is to detect the face in each of these frames. This is achieved by making use of the Haarcascade file for face detection. The Haarcascade file contains a 
number of features of the face, such as height, width and thresholds of face colors., it is constructed by using a number of positive and negative samples. For face detection, we first load the cascade file. Then pass the acquired frame to an edge detection function, which detects all the possible objects of different sizes in the frame. To reduce the amount of processing, instead of detecting objects of all possible sizes, since the face of the automobile driver occupies a large part of the image, we can specify the edge detector to detect only objects of a particular size, this size is decided based on the Haarcascade file, wherein each Haarcascade file will be designed for a particular size.

Now, the output the edge detector is stored in an array. Now, the output of the edge detector is then compared with the cascade file to identify the face in the frame. Since the cascade consists of both positive and negative samples, it is required to specify the number of failures on which an object detected should be classified as a negative sample. In our system, we set this value to 3 , which helped in achieving both accuracy as well as less processing time. The output of this module is a frame with face detected in it.

\section{Eye detection}

After detecting the face, the next step is to detect the eyes, this can be achieved by making use of the same technique used for face detection. However, to reduce the amount of processing, we mark the region of interest before trying to detect eyes. The region of interest is set by taking into account the following:

- The eyes are present only in the upper part of the face detected.

- The eyes are present a few pixels lower from the top edge of the face.
Once the region of interest is marked, the edge detection technique is applied only on the region of interest, thus reducing the amount of processing significantly. Now, we make use of the same technique as face detection for detecting the eyes by making use of Haarcascade Xml file for eyes detection.

$\mathrm{EAR}=\frac{(|p 2-p 6|+|p 3-p 5|)}{2 *|p 1-p 4|}$

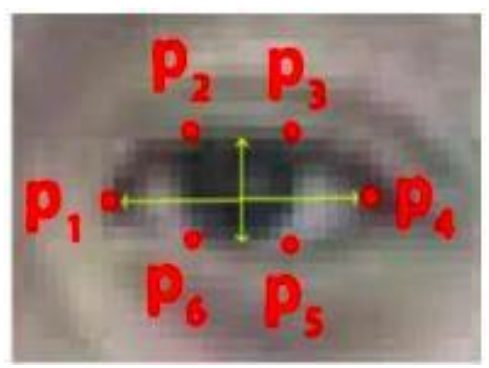

Fig 2. Landmarks of Eye in EAR

In this context, EAR values are used to detect driver's drowsiness. EAR value of left and right eyes is calculated and then average is taken. In drowsiness detector case, the Eye Aspect Ratio is monitored to check if the value falls below threshold value and also it does not increases again above the threshold value in the next frame. The above condition implies that the person has closed his/her eyes and is in a drowsy state. On the contrary, if the EAR value increases again, it implies that the person has just blinked the eye and there is no case of drowsiness.

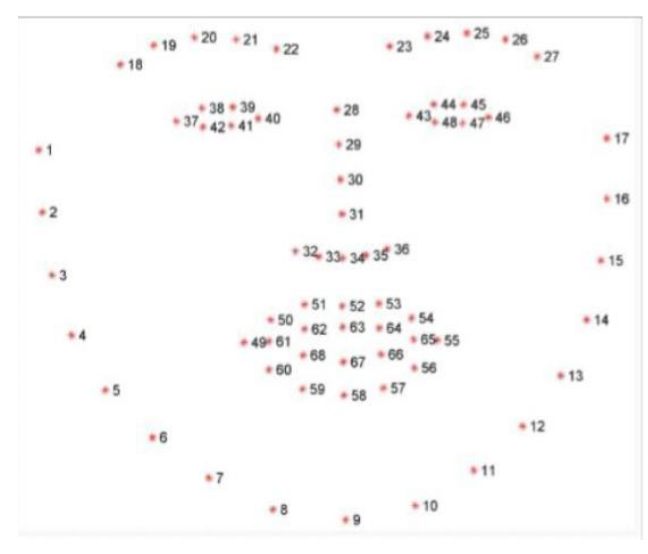

Fig 3- Facial Landmark Points according to Dlib Library 
Table 1 - Facial Landmarks

\begin{tabular}{|l|l|}
\hline \multicolumn{1}{|c|}{ Part } & \multicolumn{1}{c|}{$\begin{array}{c}\text { Landmark } \\
\text { Points }\end{array}$} \\
\hline Left Eye & {$[37-42]$} \\
\hline Right Eye & {$[43-48]$} \\
\hline
\end{tabular}

\section{Drowsiness detection}

Once the eyes are detected, the next step is to determine if the eyes are in closed or open state. This is achieved by extracting the pixel values from the eye region. After extracting, we check if these pixel values are white, if they are white then it infers that the eyes are in the open state, if the pixel values are not white then it infers that the eyes are in the closed state.

This is done for each and every frame extracted. If the eyes are detected to be closed for two seconds or a certain number of consecutive frames depending on the frame rate, then the automobile driver is detected to be drowsy. If the eyes are detected to be closed in non-consecutive frames, then $\mathrm{We}$ declare it as a blink.

If drowsiness is detected, a text message is displayed along with triggering an audio alarm. But, it was observed that the system was not able to run for an extended period of time, because the conversion of the acquired video from RGB to grayscale was occupying too much memory. To overcome this problem, instead of converting the video to grayscale, the RGB video only was used for processing. This conversion resulted in the following advantages,

- Better differentiation between colours, as it uses multichannel colours.

- Consumes very less memory.

- Capable of achieving blink detection, even when the automobile driver is wearing spectacles.

\section{Location Tracking}

After the driver has been detected as drowsy for 5 continues frames, or drivers face is not detected in the video frame captured the drivers location is send on the web server and displayed to the admin on the hosted website. The admin will also receive the email notification if any of the above conditions get true.

\section{HARDWARE \& SOFTWARE REQUIREMENTS}

\section{HARDWARE SPECIFICATION}

- Intel processor IV and above

- 2 GB RAM

- 160 GB hard disk

\section{SOFTWARE SPECIFICATION}

- Visual Studio Code

- Python 3.6 .3

- External Camera

\section{RESULT \& DISCUSSION}

This project tries to combine all major features associated with drowsiness of a driver to achieve optimum result. The features being EAR, MAR, Blink Rate and Head tilt. All the four parameters are considered simultaneously to determine whether a person is drowsy or not. Our trained haar cascade is better in identifying variations in facial structure, complexion along with identifying multiple faces in a single frame. Our haar cascade performs better in terms of computation time required to detect faces in real time scenario.

The system will detect drowsiness of the driver. Real time data will be saved on Web Portal admin will receive mail as "Driver's Drowsiness Detected". 
Location will also be traced in browser. This way admin can easily access data of the driver.

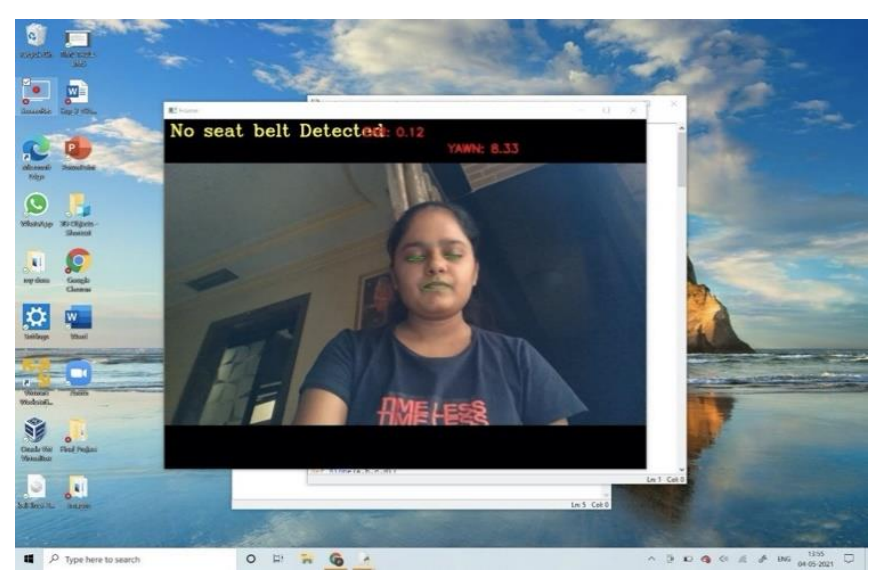

To alert the driver on the detection of drowsiness by using buzzer or alarm.
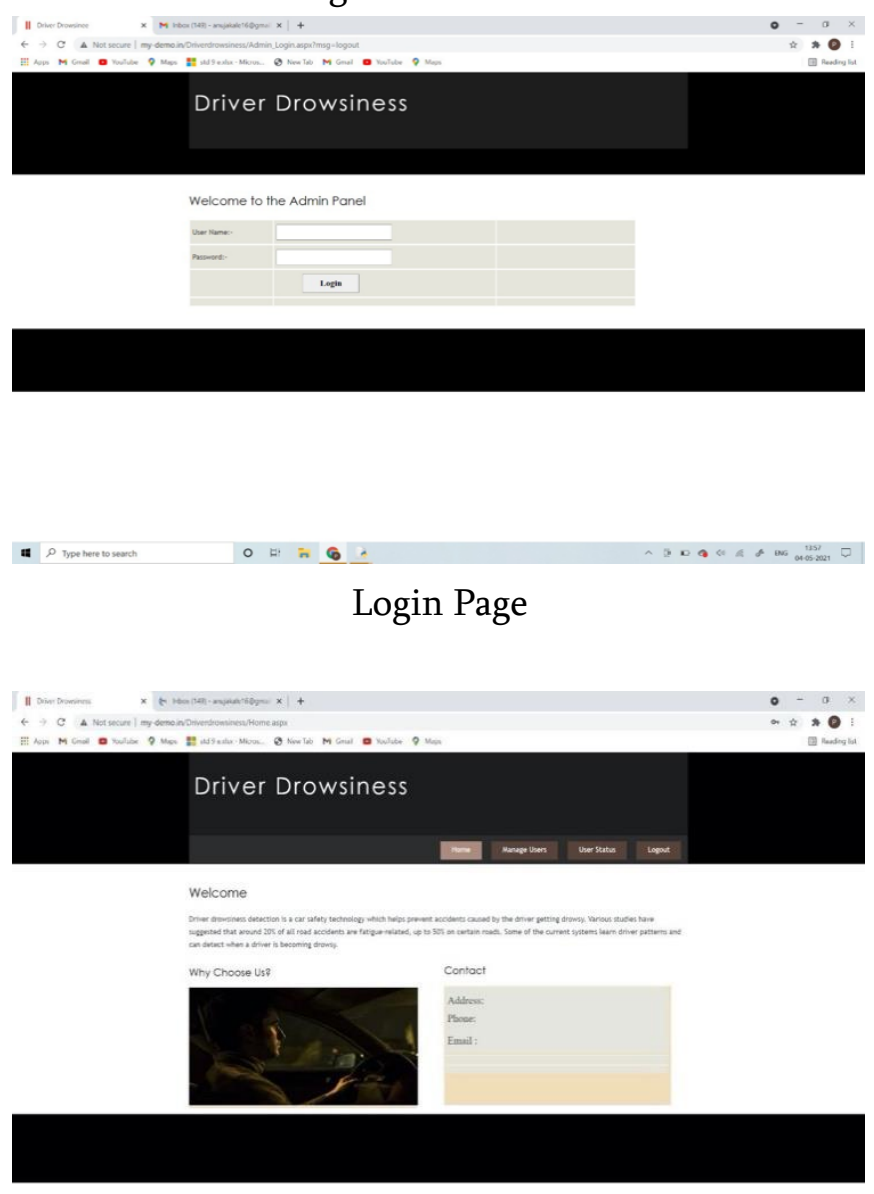

Home Page
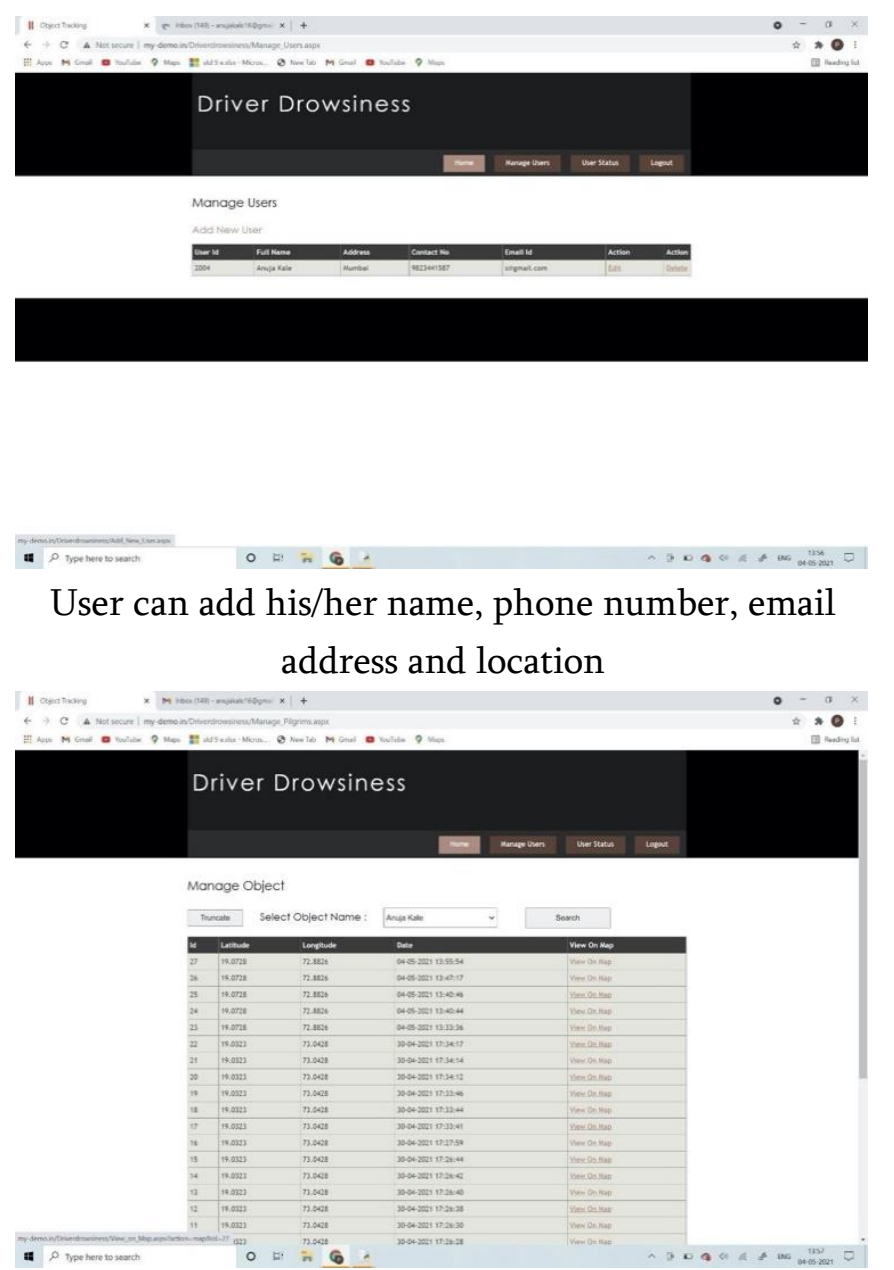

Click on View on map and you will the user's location.

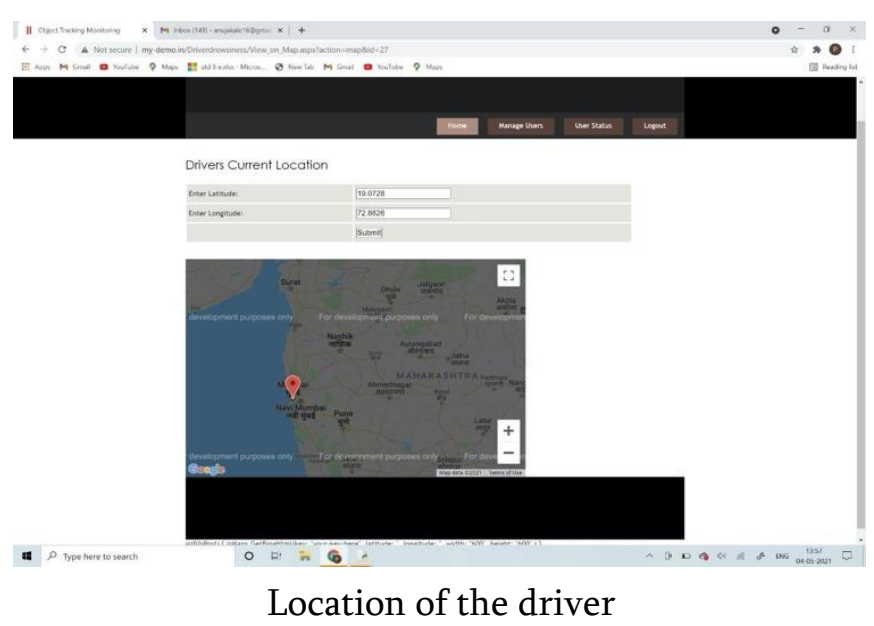

When the driver is detected drowsy, we calculate the latitude and longitude of the area in which the driver is present at that time and store the latitude and longitude co-ordinates in the database server. Using the longitude and latitude co-ordinates we can track 
the location of the driver where he is drowsy on the map.

\section{CONCLUSION}

The project intends to present a solution to alert the driver before a mishap happens. Detecting the driver drowsiness, which is one of the major cause of road accidents, will reduce deaths and injuries to a great extent. During monitoring the system is able to detect when the eyes are closed and mouth open simultaneously for too long and again and again in less period of time thus giving a buzzer sound to alert the driver. The system alerts the driver if he closes his eyes for long time which is giving information that the driver might have slept.

It completely meets the objectives and requirements of the system. The framework has achieved an unfaltering state where all the bugs have been disposed of. The framework cognizant clients who are familiar with the framework and comprehend it's focal points and the fact that it takes care of the issue of stressing out for individuals having fatigue-related issues to inform them about the drowsiness level while driving.

\section{ACKNOWLEDGEMENT}

We would like to thank Dr. Swati Sinha for giving us the opportunity to work on the project and for providing the necessary resources.

\section{REFERENCES}

[1]. C. Murukesh, Preethi Padmanabhan, Department of Electronics and Instrumentation Engineering, Velammal Engineering College, Anna University, Chennai, INDIA. "DROWSINESS DETECTION FOR DRIVERS USING COMPUTER VISION",WSEAS
Transactions on In- formation Science and Applications

[2]. Ghassan Jasim ALAnizy, Md. Jan Nordin and Mohammed M. Razooq,"AUTOMATIC DRIVER DROWSINESS DETECTION USING HAAR ALGORITHM AND SUPPORT VECTOR MACHINES TECHNIQUES"

[3]. Mehul K Dabhi, Bhavna K Pancholi, M. S. University Baroda, India, " FACE DETECTION SYSTEM BASED ON VIOLA-JONES ALGORITHM", International Journal of Science and Research (IJSR).

[4]. P. Viola and M. Jones, " RAPID OBJECT DETECTION USING BOOSTED CASCADE OF SIMPLE FEATURES”, Proceedings IEEE Conf. on Computer Vision and Pattern Recognition 2001.

[5]. G. Bradski and A. Kaehler, "LEARNING OPENCV: COMPUTER VISION WITH THE OPENCV LIBRARY" , O'Reilly Media, Inc., 2008.

[6]. Ruian Liu,et.a;, "Design of face detection and tracking system," Image and Signal Processing (CISP), 2010 3rd International Congress on , vol.4, no., pp.1840,1844, 16- 18 Oct. 2010

[7]. Xianghua Fan, et.al, "The system of face detection based on OpenCV," Control and Decision Conference (CCDC), 2012 24th Chinese , vol., no., pp.648,651, 23-25 May 2012

[8]. Parris, J., et.al, "Face and eye detection on hard datasets," Biometrics (IJCB), 2011 International Joint Conference on , vol., no., pp.1,10, 11-13 Oct. 2011

[9]. Picot, A. et.al., "On-Line Detection of Drowsiness Using Brain and Visual Information," Systems, Man and Cybernetics, Part A: Systems and Humans, IEEE Transactions on , vol.42, no.3, pp.764,775,May 2012

[10]. Xia Liu; Fengliang Xu; Fujimura, K., "Real-time eye detection and tracking for driver 
observation under various light conditions," Intelligent Vehicle Symposium, 2002. IEEE, vol.2, no., pp.344,351 vol.2, 17-21 June 2002

[11]. 11.Punit Lohani, Rohan Putta ,Gayatri N Shinde,"REAL TIME DROWSINESS DETECTION SYSTEM USING VIOLA JONES ALGORITHM ",International Journal of Computer Applications (0975 8887) Volume 95 No.8, June 2014 .

[12]. Nafis IRTIJA, Mahsius SAMI, Md Atiqur Rahman AHAD,"FATIGUE DETECTION USING FACIAL LANDMARKS",ISASE-MAICS 2018 .

[13]. C. Patel, S. A. Khan, and V. N. Patil, "RealTime Driver Drowsiness Detection System Based on Visual Information," vol. 8, no. 3, pp. 16200- 16203, 2018.

\section{Cite this article as :}

Anuja Kale, Aditya Raut, Swati Sinha, "Intelligent Drive Assistance System", International Journal of Scientific Research in Computer Science, Engineering and Information Technology (IJSRCSEIT), ISSN : 2456-3307, Volume 7 Issue 3, pp. 96-104, May-June 2021. Available at doi : https://doi.org/10.32628/CSEIT217312 Journal URL : https://ijsrcseit.com/CSEIT217312 